Gratings for Increasing

Solid-State Laser Gain and

Efficiency

A.C. Erlandson, J.A. Britten, J.D. Bonlie

April 19, 2010 
This document was prepared as an account of work sponsored by an agency of the United States government. Neither the United States government nor Lawrence Livermore National Security, LLC, nor any of their employees makes any warranty, expressed or implied, or assumes any legal liability or responsibility for the accuracy, completeness, or usefulness of any information, apparatus, product, or process disclosed, or represents that its use would not infringe privately owned rights. Reference herein to any specific commercial product, process, or service by trade name, trademark, manufacturer, or otherwise does not necessarily constitute or imply its endorsement, recommendation, or favoring by the United States government or Lawrence Livermore National Security, LLC. The views and opinions of authors expressed herein do not necessarily state or reflect those of the United States government or Lawrence Livermore National Security, LLC, and shall not be used for advertising or product endorsement purposes.

This work performed under the auspices of the U.S. Department of Energy by Lawrence Livermore National Laboratory under Contract DE-AC52-07NA27344. 
Gratings for Increasing Solid-State Laser Gain and Efficiency

A. C. Erlandson, J. A. Britten and J. D. Bonlie

Lawrence Livermore National Laboratory

April 16, 2010 


\begin{abstract}
We introduce new concepts for increasing the efficiency of solid state lasers by using gratings deposited on laser slabs or disks. The gratings improve efficiency in two ways: 1) by coupling out of the slab deleterious amplified spontaneous emission (ASE) and

2) by increasing the absorption efficiency of pump light. The gratings also serve as antireflective coatings for the extracting laser beam. To evaluate the potential for such coatings to improve laser performance, we calculated optical properties of a 2500 groove $/ \mathrm{mm}$, tantala-silica grating on a $1 \mathrm{~cm} \mathrm{x} 4 \mathrm{~cm} \times 8 \mathrm{~cm}$ titanium-doped sapphire slab and performed ray-trace calculations for ASE and pump light. Our calculations show substantial improvements in efficiency due to grating ASE-coupling properties. For example, the gratings reduce pump energy required to produce a $0.6 / \mathrm{cm}$ gain coefficient by $9 \%, 20 \%$ and $35 \%$ for pump pulse durations of $0.5 \mu \mathrm{s}, 1 \mu \mathrm{s}$ and $3 \mu \mathrm{s}$, respectively. Gratings also increase 532-nm pump-light absorption efficiency, particularly when the product slab overall absorption is small. For example, when the single-pass absorption is 1 neper, absorption efficiency increases from $66 \%$, without gratings, to $86 \%$, when gratings are used.
\end{abstract}

\title{
Introduction
}

Amplified spontaneous emission (ASE) is generated in solid-state gain media when excited ions spontaneous decay and the resulting light is amplified. As ASE propagates through the slab, it is amplified at the expense of gain and stored energy that could otherwise be used to amplify the laser beam. ${ }^{1}$ Since the greatest reduction in gain coefficient and stored-energy density occurs at the ends of the longest gain paths, ASE preferentially reduces gain at the edges and corners of laser slabs, causing gain nonuniformity. ASE effects on gain and gain uniformity can be mitigated by increasing injected energy and by tailoring the injected fluence distribution to produce uniform output fluence. These methods add cost and complexity to large solid-state laser systems, however.

ASE internal to laser slabs is difficult to control because it radiates from the entire pumped volume over broad ranges of angle and wavelength. Currently, ASE losses are kept to acceptable levels by limiting slab internal gain, by using edge claddings that absorb ASE and by delivering pump energy in pulses that are much shorter than the radiative lifetime, thereby allowing little time for decay to occur. However, these methods place constraints on slab size and on slab gain and can increase the cost of the pump system.

Another important loss mechanism in solid-state amplifiers is the leakage of pump light through the laser slabs. Such losses are represented by $1-\eta_{\mathrm{abs}}$, where $\eta_{\mathrm{abs}}$ is the fraction of pump-light power incident on the gain medium that is absorbed. $\eta_{\text {abs }}$ depends on the product of the absorbing ion density and path length and can be increased by increasing either or both parameters. Often, however, ion density and/or path length must remain relatively low due to physical or design constraints. For example, ion density must remain sufficiently low to enable crystal growth to occur properly, to limit deleterious 
interactions between ions (e.g., the "concentration quenching" that occurs with $\mathrm{Nd}^{3+}$ ions), to limit absorption of the amplified beam by ground-state ions (e.g., in quasi-three-level systems such as $\mathrm{Yb}^{3+}$ ), or to achieve the desired value for thermal conductivity. Increases in the path length for pump light can also be limited. For example, face-pumped slab lasers operating at high average power can be made thicker, but only to the point at which thermal stress approaches the yield stress. Alternatively, several slabs might be stacked together to increase the path length for pump light, but this method complicates the amplifier design and makes uniform and efficient pumping more difficult to achieve.

In this report we describe our assessment of the feasibility of using diffraction gratings on laser slabs to reduce ASE decay losses and to increase pump-light absorption efficiency. We begin by reviewing basic concepts and show how desired effects can be achieved over certain ranges of grating groove density. We present calculation results showing the optical properties of gratings that are required for reducing ASE losses. We describe in detail a grating for improving the performance of titanium-doped sapphire slabs. We finish by presenting ray-trace code predictions for ASE decay rates, both with and without gratings.

\section{Background information and concepts}

In slabs having two dimensions (e.g., width and length) much greater than the third dimension (thickness), most ASE reflects back and forth between the two large, facing surfaces. Rays with angle of incidence greater than critical angle are totally reflected, while rays with angle of incidence less than the critical angle are only partially reflected. The critical angle, $\theta_{\mathrm{c}}$, is given by

$$
\theta_{c}=\sin ^{-1}\left(n_{0} / n\right)
$$

where $\mathrm{n}$ is the refractive index of the laser slab and $\mathrm{n}_{0}$ is the refractive index of the medium outside the slab. The fraction of spontaneously-emitted light that is trapped by total-internal reflection (TIR) depends on the refractive index and is $\sim 75 \%$ for phosphate glass with $\mathrm{n} \sim 1.52$.

ASE that is trapped by TIR can build up to high intensity since reflectance at surfaces is $100 \%$ and trapped light is amplified on each pass through the gain medium. For a gain medium with a long pumped dimension L across the aperture, the path length $\mathbf{s}$ for TIR rays undergoing one or more reflections is given by

$$
\mathrm{s}=\frac{\mathrm{L}}{\sin \theta_{\mathrm{i}}}
$$

where $\theta_{\mathrm{i}}$ is the angle of incidence at the large slab faces. See Figure 1a. Of the rays that are totally reflected, those at the critical angle have the longest ray paths and the highest gain. Their overall path length is $\mathrm{nL}$ and their gain in Nepers, for an average gain coefficient $\alpha$, is n $\alpha \mathrm{L}$. ASE decay rates are often characterized as functions of $\alpha \mathrm{L}$ or noL. 
When gratings are present, ASE arriving at the slab surfaces can have significant transmittance even when angles of incidence are greater than the critical angle. Additionally, gratings can reflect light onto the opposite side of the slab where it can also be transmitted, provided that the angle of incidence is below the critical angle (or the opposite side also has a grating). Both channels are illustrated in Figure 1b. Provided that net gain (reflectance $\mathrm{x}$ gain) on each round trip through the slab is less than unity, ASE intensity does not grow and ASE decay losses become small.

(a)

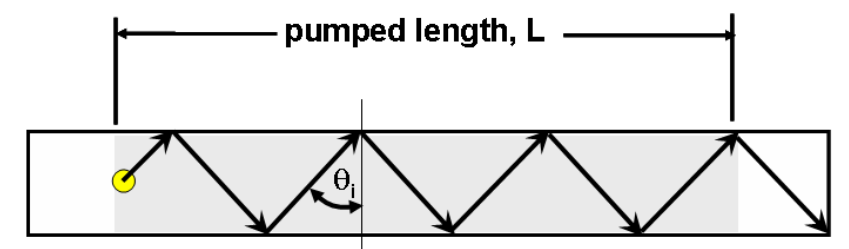

(b)

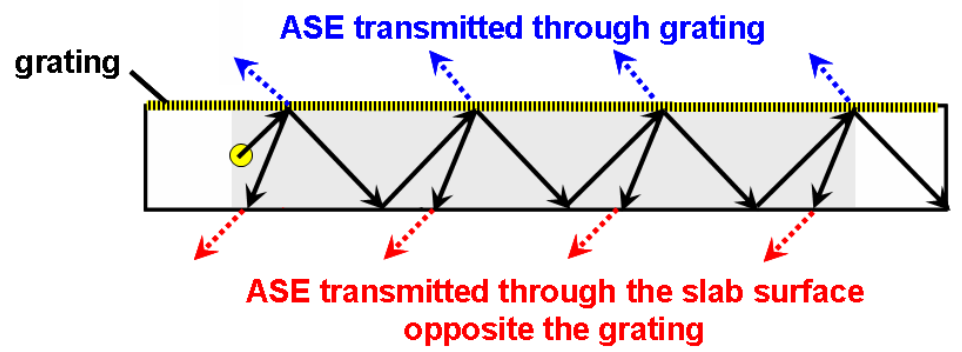

Figure 1. (a) Without gratings, ASE trapped by TIR is amplified, without loss, on round trip through the slab. (b) With gratings, ASE is no longer trapped by TIR and transmission losses partially offset gain. ASE is coupled out of the slab either directly, by transmission through the grating, or indirectly, by transmission through the surface opposite the grating following diffraction at an angle less than the critical angle.

Diffraction gratings also offer potential for increasing solid-state laser efficiency by enhancing pump-light absorption efficiency. Gratings enhance absorption efficiency by diffracting pump light into paths having greater length through the gain medium than simply-refracted rays. Gratings can diffract pump light either in transmission, as shown in Figure 2a, or in reflection, as shown in Figure 2b. Note also that gratings can increase pump-light intensity. Intensity is increased when pump light is diffracting at steep angles, so that the width of the diffracted beam is smaller than the width of the initial pump beam. Pump-light intensity is also increased when diffracted light that is not absorbed after one pass is reflected back through the slab for an additional pass, as occurs when the gain medium is weakly absorbing and the diffraction angle is greater than the critical angle for TIR. Pump-light intensification can be especially beneficial for quasi-three-level lasers, which often require some degree of bleaching to overcome ground-state absorption. 
(a)

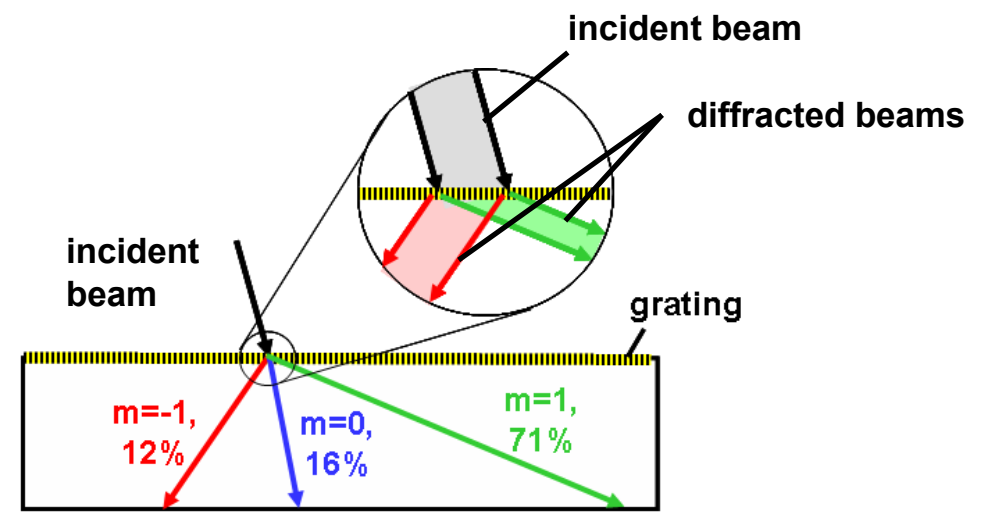

(b)

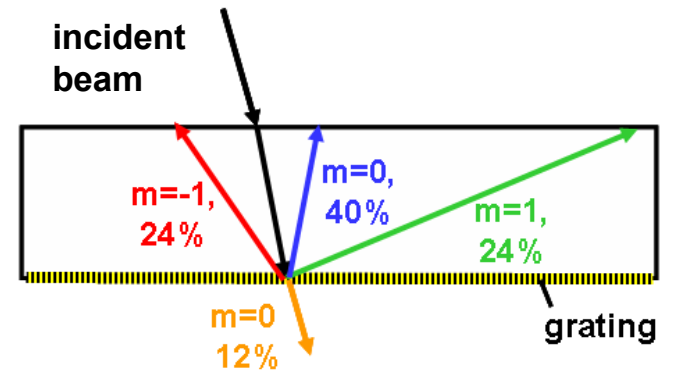

Figure 2. (a) Gratings diffract incident pump light into various transmission orders, for which path lengths for some can be significantly greater than for simply-refracted light. Diffracted beams can have smaller beam widths and higher intensity than the incident beam, as indicated by the inset. (b) Gratings can also diffract light into various reflection orders, $m$ '.

\section{Criteria for values of grating groove separation}

We have calculated ranges of values for grating groove separation over which it is possible to achieve desired grating properties. The starting point for our calculation was the grating equation for transmitted rays,

$$
\mathrm{n} \sin \theta_{\mathrm{i}}+\mathrm{n}_{0} \sin \theta_{\mathrm{t}}=\frac{\mathrm{m}_{\mathrm{t}} \lambda}{\mathrm{d}}
$$

and the grating equation for internally reflected rays,

$$
\sin \theta_{i}+\sin \theta_{r}=\frac{m_{r} \lambda}{n d}
$$

where $\theta_{\mathrm{t}}$ is the transmitted angle, $\theta_{\mathrm{r}}$ is the reflected angle, $\mathrm{m}_{\mathrm{t}}$ is the transmission order, $\mathrm{m}_{\mathrm{r}}$ is the reflection order, $\lambda_{\text {ASE }}$ and $\lambda_{\text {laser }}$ are wavelengths in vacuum, and $d$ is the grating groove separation. Results of these calculations are summarized below. 
1. To ensure possible $m_{t}=1$ transmittance for all internal ASE rays that are incident on the grating at angles greater than the critical angle,

$$
\frac{\lambda_{\mathrm{ASE}}}{\mathrm{n}+1} \leq \mathrm{d} \leq \frac{\lambda_{\mathrm{ASE}}}{\mathrm{n}-1}
$$

2. to eliminate the possibility of closed paths formed by $\mathrm{m}_{\mathrm{r}}=1$ reflections at the Littrow angle inside the slab, which pose a parasitic risk,

$$
\mathrm{d}>\frac{\lambda_{\mathrm{ASE}}}{2 \mathrm{n}_{0}}
$$

3. to eliminate transmitted orders, other than the zeroth order, for the extracting laser beam,

$$
\mathrm{d}<\frac{\lambda_{\text {laser }}}{\mathrm{n}_{0}}
$$

Eliminating the non-zero orders is important for achieving high transmission efficiency for the extracting laser beam. Similarly,

4. to eliminate reflected orders, other than the zeroth order, for the extracting laser beam,

$$
\mathrm{d}<\frac{\lambda_{\text {laser }}}{\mathrm{n}}
$$

5. to ensure that pump light that is incident at near normal incidence has $\mathrm{m}_{\mathrm{t}}=1$ transmitted and $\mathrm{m}_{\mathrm{r}}=1$ reflected orders at angles greater than the critical angle,

$$
\mathrm{d}<\frac{\lambda_{\text {pump }}}{\mathrm{n}_{0}}
$$

Meeting this requirement ensures that diffracted pump light that passes through the slab once can undergo total internal reflection and pass through the slab a second time, thereby increasing the absorption path length.

The grating equations used to derive the five criteria above apply to light that is incident in a plane perpendicular to the grating. Criteria for rays in other planes remain to be developed.

As a first test of the feasibility of using gratings to reduce ASE and to increase pumping efficiency, the five criteria above were applied to four different gain media: titaniumdoped sapphire, Nd:YAG, Nd:phosphate glass, and Yb:S-FAP. For all four media, there is a range of values for the groove separation over which all five criteria are met. Table 1 
gives parameters used for evaluating the four different gain media. Table 2 gives calculated ranges of grating groove separation that meet the five criteria.

Table 1. Parameters used for calculating groove separations that can achieve desirable grating properties.

\begin{tabular}{|c|c|c|c|c|}
\hline parameter & Ti:Sa & Nd:YAG & Nd:Glass & Yb:S-FAP \\
\hline $\mathrm{n}_{\mathrm{s}}$ & 1.76 & 1.82 & 1.52 & 1.625 \\
\hline $\mathrm{n}_{0}$ & 1 & 1 & 1 & 1 \\
\hline$\lambda_{\text {ASE,min }}(\mathrm{nm})$ & 700 & 1060 & 1040 & 1043 \\
\hline$\lambda_{\text {ASE,max }}(\mathrm{nm})$ & 900 & 1070 & 1070 & 1051 \\
\hline$\lambda_{\text {laser }}(\mathrm{nm})$ & 800 & 1064 & 1053 & 1047 \\
\hline$\lambda_{\text {pump }}(\mathrm{nm})$ & 527 & 808 & 808 & 900 \\
\hline
\end{tabular}

Table 2. Ranges of grating groove spacing, $d$, required for achieving desirable grating properties. Values are given in $\mathbf{n m}$.

\begin{tabular}{|cc|c|c|c|c|}
\hline Criterion \# & & Ti:Sa & Nd:YAG & Nd:Glass & Yb:S-FAP \\
\hline \multirow{2}{*}{1} & $\mathrm{~d} \geq$ & 326 & 379 & 425 & 400 \\
& $\mathrm{~d} \leq$ & 921 & 1293 & 2000 & 1669 \\
\hline 2 & $\mathrm{~d}>$ & 450 & 535 & 535 & 526 \\
\hline 3 & $\mathrm{~d}<$ & 800 & 1064 & 1053 & 1047 \\
\hline 4 & $\mathrm{~d}<$ & 455 & 585 & 693 & 644 \\
\hline 5 & $\mathrm{~d}<$ & 527 & 808 & 808 & 900 \\
\hline \multirow{2}{*}{ Summary } & $\mathrm{d} \geq$ & 450 & 535 & 535 & 525.5 \\
& $\mathrm{~d}<$ & 455 & 585 & 693 & 644 \\
\hline
\end{tabular}

\section{Criteria for grating optical properties}

To reduce ASE losses, it is desirable for gratings to have reflectance values that are smaller than the inverse of the two-pass small-signal gain. When this requirement is met, the net gain for one or more passes is less than unity and ASE does not grow as it propagates across the slab. To determine reflectance requirements, small-signal gain was calculated for TIR-trapped rays propagating across the slab. Figure 3 below shows smallsignal gain vs. angle of incidence for a slab with refractive index of 1.76 (for Ti-doped sapphire) and an ASE parameter noL of 6, which is a typical value of $n \alpha \mathrm{L}$ for large laser systems. ${ }^{2}$ As can be seen, rays close to the critical angle have the greatest gain, while rays that are more than 15-20 degrees above the critical angle have substantially lower gain. Rays near the critical angle make more passes through the slab and have greater overall path lengths. 


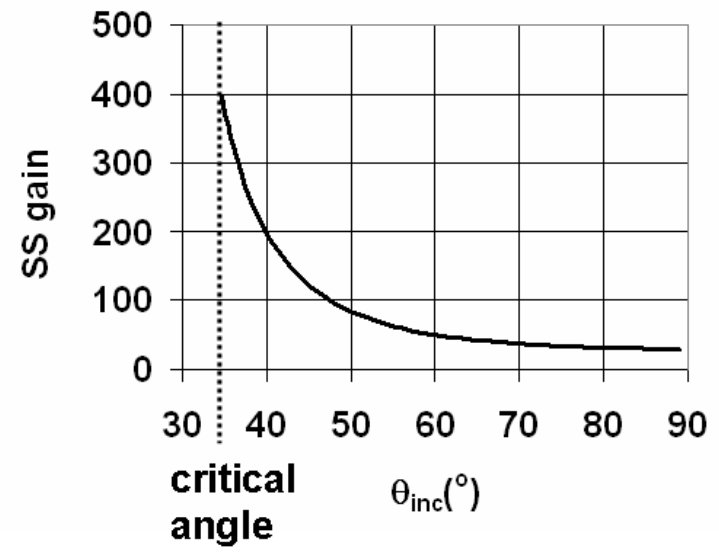

Figure 3. Small-signal gain plotted vs. angle of incidence for ASE trapped by total internal reflection (TIR) within a laser slab. For this plot, the refractive index is 1.76 (same as for Ti-doped sapphire) and the ASE parameter $n \alpha L$ is 6 . The TIR-trapped rays that have the highest gain are those closest to the critical angle.

Figure 4 presents a contour plot for reflectance values that give a net gain of unity for rays at the critical angle. The contours are plotted versus slab aspect ratio L/t and ASE parameter n $\alpha \mathrm{L}$, where $\mathrm{L}$ is the pumped length of the slab and $\mathrm{t}$ is the slab thickness. For $\mathrm{L} / \mathrm{t}=15$ and $\mathrm{n} \alpha \mathrm{L}=6$, which correspond to the amplifiers in the National Ignition Facility, a reflectance value of $\sim 0.55$ just offsets the two-pass small-signal gain of $\sim 1.33^{2}=1.77$. When either $\alpha$ is increased (i.e., the design point is moved upwards on the contour plot) or $t$ is increased (i.e., the design point is moved to the left on the contour plot), two-pass gain is increased and smaller values of reflectance are needed to achieve unity net gain. Reflectance requirements for reducing ASE become more relaxed as slab thickness and gain coefficient become smaller. While this analysis is oversimplified for estimating reflectance requirements for gratings, because gratings diffract rays into several orders, it is instructive to know that reflectance values in the mid-range between 0 and 1 are adequate for keeping ASE from growing. We used this result as a guide for designing our first gratings. 


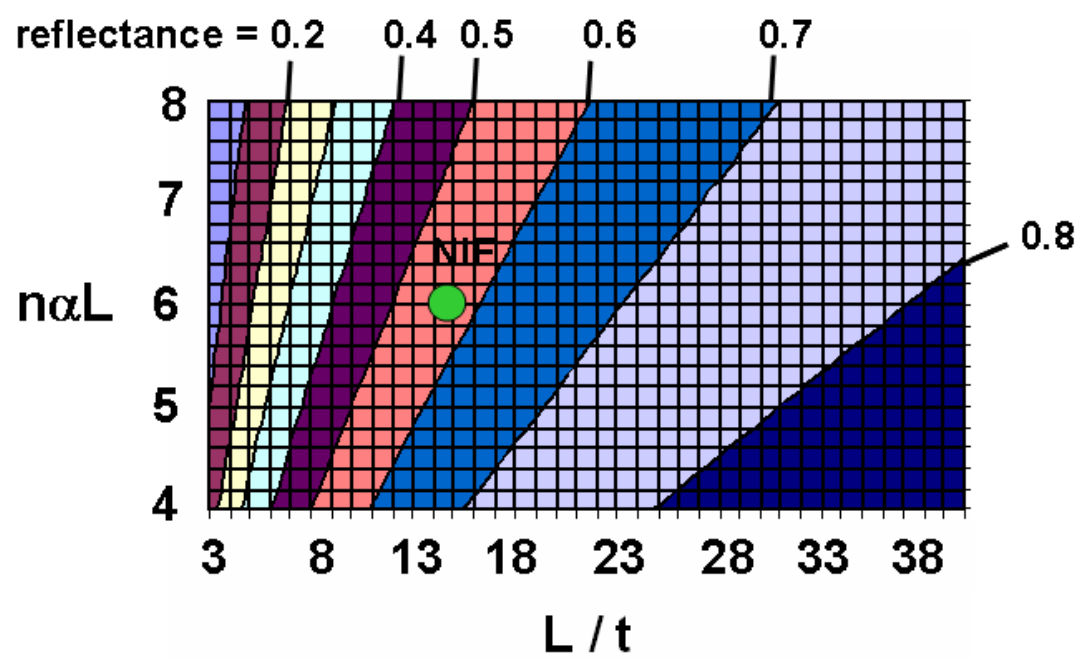

Figure 4. A contour plot for reflectance values that give unity net gain at the critical angle for total-internal reflection. Axes are slab aspect ratio L/t and ASE parameter $n \alpha L$. For reference, NIF slabs have an $L / t$ ratio of about 15 and an naL value of about 6 , as indicated by the green dot.

\section{Grating Design}

We have analyzed in detail a grating designed for use on titanium-doped sapphire slabs, shown schematically in Figure 5. To ensure that ASE is coupled from the slab into the grating structure efficiently, the bottom coating layer in the dielectric stack uses $\mathrm{Ta}_{2} \mathrm{O}_{5}$, a material with a higher refractive index $(\sim 2.2)$ than the sapphire substrate. Atop this layer are layers of $\mathrm{Ta}_{2} \mathrm{O}_{5}$ and $\mathrm{SiO}_{2}$ with thicknesses of $350 \mathrm{~nm}$ and $120 \mathrm{~nm}$, respectively. $\mathrm{Ta}_{2} \mathrm{O}_{5}$ and $\mathrm{SiO}_{2}$ are commonly-used coating materials and neither absorbs substantially at either the pump wavelength or the extraction wavelength, which are $527 \mathrm{~nm}$ and 800 $\mathrm{nm}$, respectively. The groove separation of $400 \mathrm{~nm}$ chosen fails to meet criterion $\# 2$ in Table 2 above, and so is not ideal. It is possible that failure to meet this criterion causes increased ASE at high values of the gain coefficient, due to Littrow reflections causing closed gain paths. The identification of criterion \#2 was motivated as a result of our analysis. 


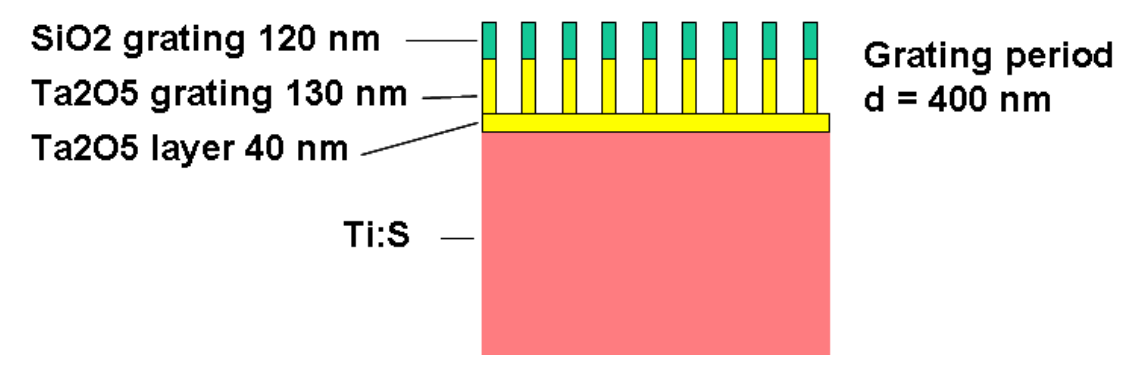

Figure 5. Grating design we developed for use on Ti-doped sapphire slabs. This design has layers of $\mathrm{Ta}_{2} \mathrm{O}_{5}$ and $\mathrm{SiO}_{2}$ and a groove separation of $400 \mathrm{~nm}$.

Gsolver, a commercial software product based on rigorous coupled wave theory, was used to calculate optical properties of the grating. Figure 6 shows calculated values for overall transmittance, $0^{\text {th }}$-order reflectance, and $1^{\text {st }}$-order reflectance, for s- and $\mathrm{p}$ polarized light, at $800 \mathrm{~nm}$. Results are plotted vs. angle of incidence, $\theta_{\text {inc }}$, for different angles of azimuth, $\phi$, relative to the grating direction. See Figure 7. Four different values of $\phi--0^{\circ}, 22.5^{\circ}, 45^{\circ}$, and $67.5^{\circ}-$ - span the range over which variations in grating properties are significant. Grating properties vary little between $67.5^{\circ}$ and $90^{\circ}$. Transmittance and reflectance values averaged over azimuth angles are also plotted, as are transmittance and reflectance values for bare slabs. Overall transmittance is the sum of $0^{\text {th }}$-order and $1^{\text {st }}$-order transmittance. The grating has no orders greater than \pm 1 , in either transmission or reflection, at $800 \mathrm{~nm}$.

Figure 8 gives another representation of the calculated optical properties of gratings, using contour plots. Contour plots for overall transmittance, $0^{\text {th }}$-order reflectance, and $1^{\text {st }}$ order reflectance, for $\mathrm{s}$ and $\mathrm{p}$ polarizations, are plotted versus $\theta_{\text {inc }}$ and $\phi$.

It is useful to compare optical properties of gratings with optical properties of bare slabs. As can be seen in Figures 6a and 6b, overall transmittance for the s and p polarizations, either with the grating or without it, tends to be high below the critical angle $\left(\sim 34.62^{\circ}\right)$ and low above the critical angle. For the bare surface, transmittance above the critical angle is zero. For the grating surface, however, transmittance above the critical angle is nonzero, with the highest values attained at small angles of incidence and small angles of azimuth. Grating enhancements in transmittance are greater for p-polarized light than for s-polarized light. High transmittance is desirable for reducing ASE intensities. Enhancements in transmittance produced by the gratings are rather modest, however.

Figures $6 \mathrm{c}$ and $6 \mathrm{~d}$ show that, as expected, 0 th-order reflectance tends to be low below the critical angle and high above. The $0^{\text {th }}$-order reflectance above the critical angle tends to be substantially lower than the bare-slab reflectance, however, particularly for azimuth angles less than $45-60^{\circ}$ and for angles of incidence close to the critical angle. 
Key

$$
\begin{array}{ll}
\hline & \text { bare slab } \\
& \text { with grating, averaged over } \phi \\
--- & \phi=67.5^{\circ} \\
--- & \phi=45^{\circ} \\
---, \phi=22.5^{\circ} & \\
--- & \phi=0^{\circ}
\end{array}
$$

s polarization
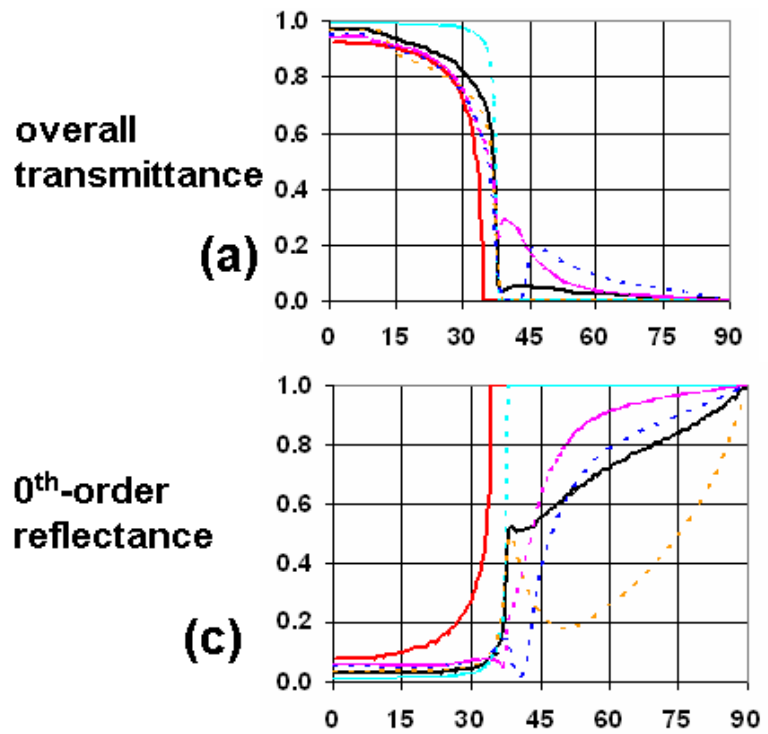

$1^{\text {st }}$-order reflectance

(e)

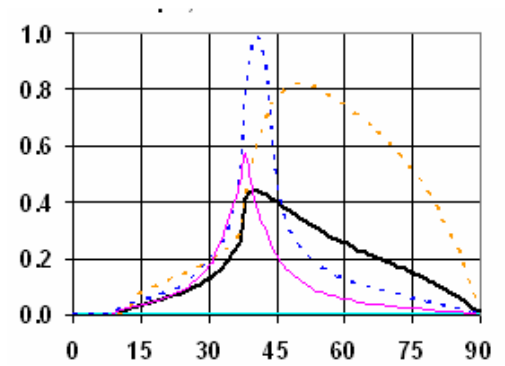

(b)

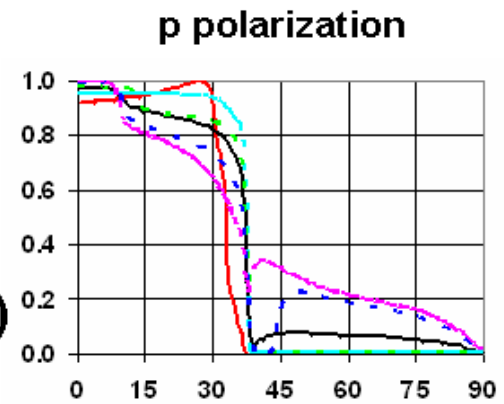

(d)

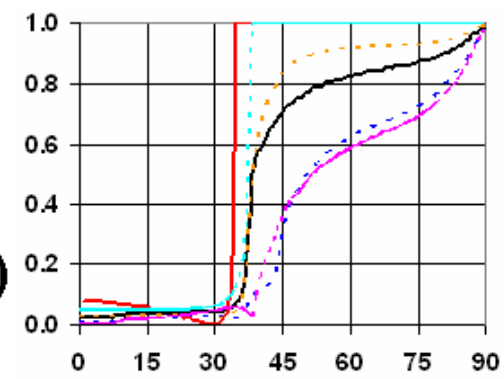

(f)

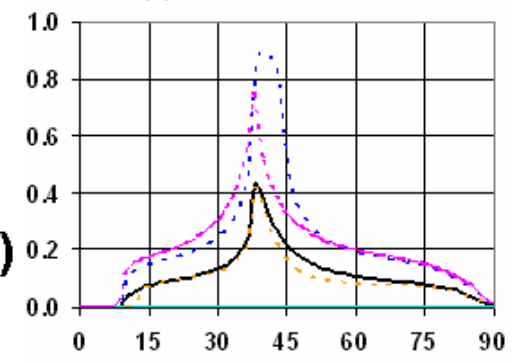

$\theta_{\text {inc }}$

Figure 6. Calculated values at $800 \mathrm{~nm}$ for overall transmittance, $0^{\text {th }}$-order reflectance, and $1^{\text {st }}$-order reflectance, for $s$ and $p$ polarizations, plotted versus angle of incidence, $\theta_{\text {inc. }}$ Results are given for four different angles of azimuth and for the average over all angles of azimuth. Overall transmittance and reflectance for a bare slab are also provided, for comparison. 


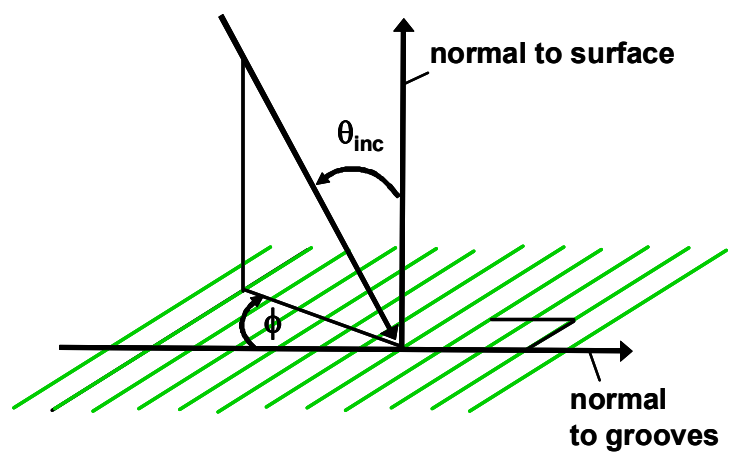

Figure 7. Sketch showing angle coordinates $\theta_{\text {inc }}$ and $\phi$ used to specify the direction of incident light rays with respect to the grating.

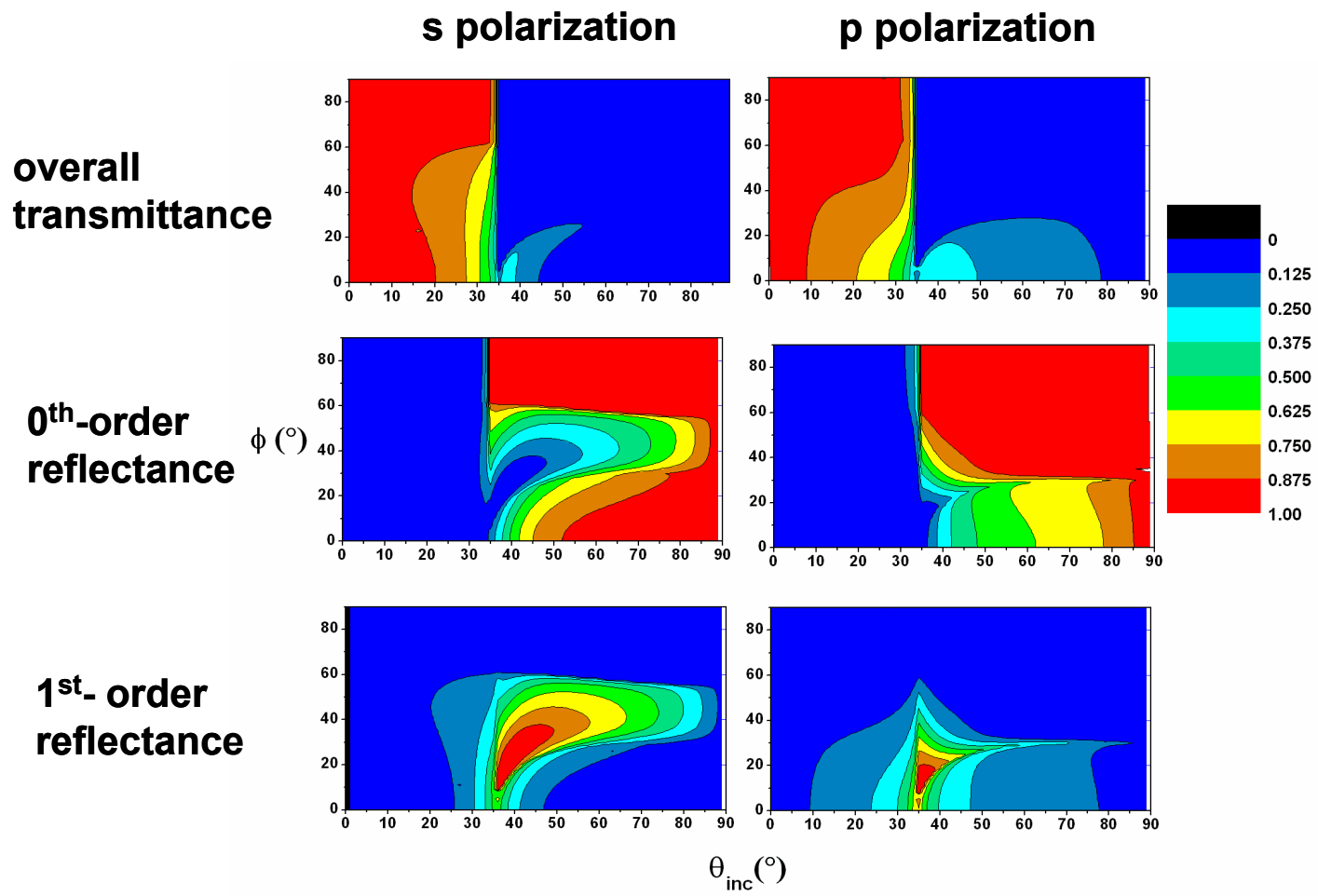

Figure 8. Contour plots showing calculated transmittance and reflectance values at $800 \mathrm{~nm}$ for the grating design illustrated in Figure 5. The horizontal axes are the angle of incidence, $\theta_{\text {inc }}$, and the azimuth angle, $\phi$, measured relative to the direction of the grating grooves. Angles are for rays incident on the grating from within the Ti-doped laser slab.

Figures $6 \mathrm{e}$ and $6 \mathrm{f}$ show $1^{\text {st }}$-order reflectance for the $\mathrm{s}$ and $\mathrm{p}$ polarizations for the grating surfaces. $1^{\text {st }}$-order reflectance is highest at incident angles slightly above the critical angle, for azimuth angles of $10-50^{\circ}$ for the s polarization, and for azimuth angles of $10-30^{\circ}$ for the p polarization. The range of incidence angles over which $1^{\text {st }}$-order reflectance is high is greater for the $\mathrm{s}$ polarization than for the $\mathrm{p}$ polarization. High $1^{\mathrm{st}}$-order reflectance 
above the critical angle, as is achieved with this grating design, is desirable for coupling ASE through the slab surface opposite the grating, as illustrated in Figure 1b. This effect occurs because $1^{\text {st }}$-order reflections for incident angles above the critical angle have diffraction angles below the critical angle, for which transmittance through the opposite slab surface is non-zero and can be high.

\section{Ray-Trace Modeling}

ASE decay rates were modeled using ASEcalc, a ray-trace code that was developed at LLNL in the early 1990's for calculating ASE decay rates in Nd:glass slabs. In its original version, ASEcalc uses Monte-Carlo methods to generate rays, with probabilities proportional to the local excited-state ion concentration. Rays are generated with a uniform angular distribution, which is appropriate for isotropic gain media such as $\mathrm{Nd}$ :glass. Ray power is tracked using its logarithm, so that gain can be modeled by simply adding the gain coefficient integrated along the ray path length. Rays incident on slab surfaces greater than the critical angle for total internal reflection (TIR) have unity reflectance while rays incident below the critical angle have reflectance equal to the average Fresnel reflectance for $\mathrm{s}$ and $\mathrm{p}$ polarizations. The instantaneous overall decay rate is calculated by summing the power of rays exiting the slab. TIR rays, which dominate ASE losses, exit the slab at the edges, which are assumed to be perfect absorbers. A key output of ASEcalc is a value for the parameter $\mathrm{M}_{\mathrm{ASE}}$, which is the multiplicative factor that gives the slab-averaged ASE decay rate when multiplied by the spontaneous emission rate.

We modified ASEcalc for the task of modeling titanium-doped sapphire laser slabs, with and without gratings. Sapphire is a negative uniaxial crystal in which light polarized parallel to the extraordinary (c) axis has about twice the stimulated-emission cross section as light polarized in an orthogonal direction. ${ }^{3}$ Therefore, we added polarization-tracking capabilities, with rays split into ordinary and extraordinary components in the gain medium and into s- and p-polarization components at the slab surfaces and gratings. These changes allowed us to use ray-generation rates that are proportional to polarization-dependent spontaneous-emission decay rates and to allow gain coefficients to be different along the two polarizations. Reflectance values for gratings, which were calculated using Gsolver, were stored in look-up tables. Ray polarization components were combined assuming incoherence, which is appropriate for broad-bandwidth ASE. ASE decay rates were averaged over the titanium emission spectrum. To simplify calculations, the spectral properties of the gratings at $800 \mathrm{~nm}$ were used over the entire emission band. Also, the extraordinary axis was oriented in the direction perpendicular to the large slab surfaces.

Figure 9 shows $\mathrm{M}_{\mathrm{ASE}} \mathrm{vs.} \mathrm{n} \alpha \mathrm{L}$ for a titanium-doped sapphire laser slab measuring $8 \mathrm{~cm} \mathrm{x}$ $16 \mathrm{~cm} \times 1 \mathrm{~cm}$, for four different combinations of grating surfaces and bare surfaces. In all four cases, $\mathrm{M}_{\mathrm{ASE}}$ increases as $n \alpha \mathrm{L}$ increases. Also, $\mathrm{M}_{\mathrm{ASE}}$ values were found to depend on the number of gratings used and the orientation of their grooves. In order of decreasing $\mathrm{M}_{\mathrm{ASE}}$, the four cases we modeled were: (a) bare on both sides; (b) bare on one side and gratings on the other side, with grooves parallel to the long slab dimension; (c) bare on 
one side and gratings on the other side, with grooves perpendicular to the long slab dimension; and (d) gratings on both sides, with grooves parallel to the long slab dimension on one side and with grooves perpendicular to the long slab dimension on the other side. Comparing curves (a) and (d), we find that using gratings on both sides reduces ASE decay rates by $20-40 \%$, depending on the value of naL. Using gratings on one side reduces ASE decay rates nearly as much as using gratings on one side, provided that the grooves are oriented perpendicular to the long slab dimension.

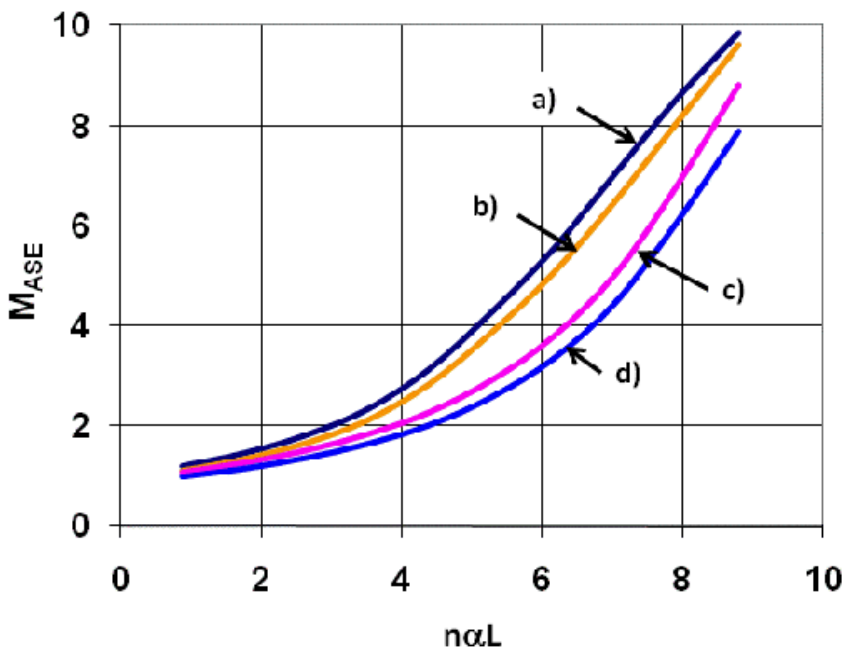

Figure 9. $M_{A S E}$ plotted vs. naL with a) two bare surfaces; b) with gratings on one side, with grooves parallel to the long slab dimension; c) with gratings on one side, with grooves perpendicular to the long slab dimension; and d) with gratings on both surfaces, with grooves parallel to the long slab dimension on one side and grooves perpendicular to the long slab dimension on the other.

\section{Performance Modeling}

We calculated gain of a $1 \mathrm{~cm}$ x $8 \mathrm{~cm}$ x $16 \mathrm{~cm}$ titanium-doped laser slab pumped by square pump pulses of various durations. In our calculations, the pump rate was a fixed value corresponding to the pump energy divided by the pump duration (and accounting for the quantum defect) and the decay rate was the inverse of the radiative lifetime ( $3 \mu \mathrm{s}$ ) multiplied by $\mathrm{M}_{\mathrm{ASE}}$, for which values corresponded to the curves shown in Figure 9, above. We modeled the case with both surfaces bare and the case with both surfaces having gratings that are orthogonal to each other.

Figure 10 shows calculated gain coefficient vs. pump-pulse energy for three of the pumppulse durations modeled. For 20-ns pump pulse duration, decay losses are small and there is little difference in the energy required to produce a specified gain coefficient, between the cases with and without gratings. For $2-\mu$ s and $4-\mu$ s pump pulse durations, decay losses are significant, greater pump-pulse energies are required and differences between the cases with and without gratings appear. 


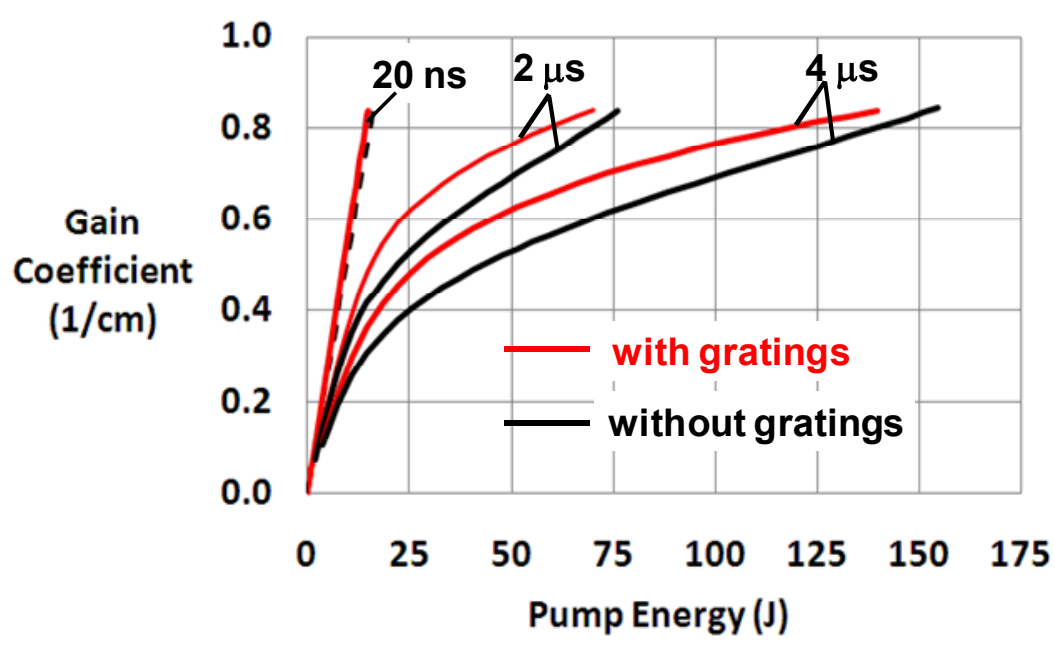

Figure 10. Calculated gain coefficient vs. optical pump energy, with gratings and without gratings, for square pulse durations of $20 \mathrm{~ns}, 2 \mu \mathrm{s}$, and $4 \mu \mathrm{s}$.

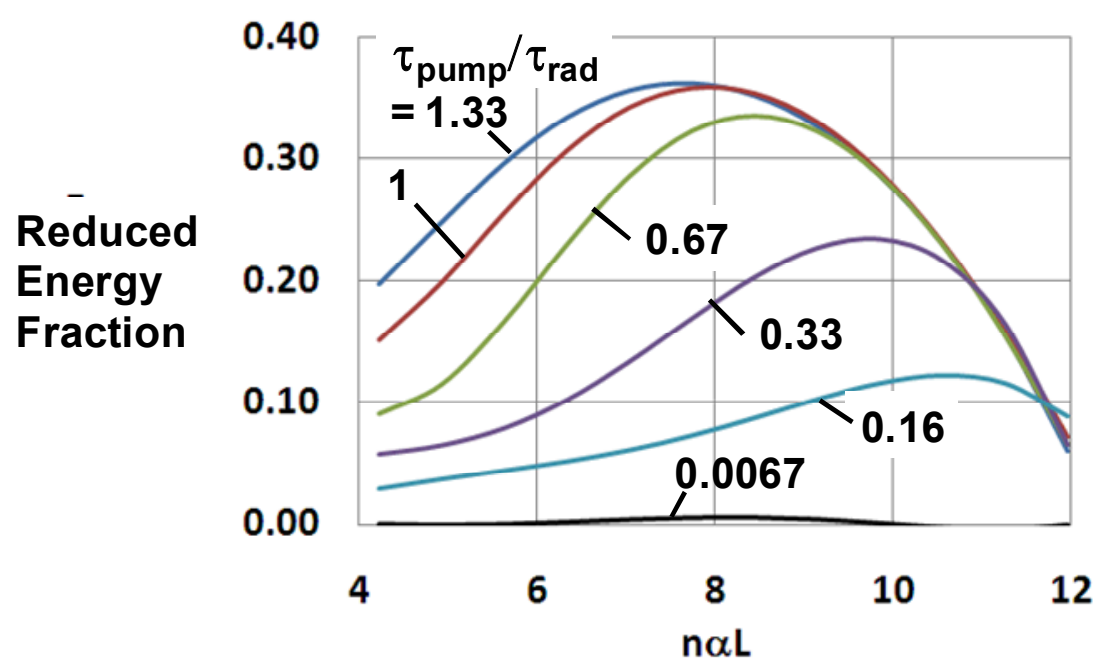

Figure 11. Reduced energy fraction vs. gain coefficient for square pump pulses of duration $20 \mathrm{~ns}, 0.5 \mu \mathrm{s}, 1 \mu \mathrm{s}, 2 \mu \mathrm{s}, 3 \mu \mathrm{s}$, and $4 \mu \mathrm{s}$. Reduced energy fraction is the reduction in optical pump energy with gratings, relative to the optical pump energy without gratings, for a specified gain coefficient.

Figure 11 shows the reduction in absorbed pump energy needed to achieve a specified gain when gratings are used. For this plot, we show the pump-pulse duration normalized with respect to the radiative lifetime, and gain expressed as the ASE parameter noL. Reduced energy fraction is 1 - pump energy with gratings / pump energy without gratings. Reduced energy fraction is small at low values of $n \alpha \mathrm{L}$, where $\mathrm{M}_{\mathrm{ASE}}$ is small and about the same, with or without gratings. See Figure 9. For all pump-pulse durations, reduced energy fraction increases with $n \alpha \mathrm{L}$ until a maximum value is achieved, after which it falls. The longer pump-pulse durations have greater maximum reduced energy fractions, scaling up to $\sim 35 \%$ for pulse durations equal to or greater than the radiative 
lifetime. Further, the value of $\alpha \mathrm{L}$ at which the maximum reduced energy fraction is achieved falls as pump-pulse duration is increased. The reason for the fall in reduced energy fraction as n $\alpha \mathrm{L}$ is not understood. However, it is plausible that the gratings themselves set up closed-loop paths that actually enhance ASE and reduce efficiency, particularly for high values of gain. This possibility remains to be explored in the future.

To evaluate the feasibility of using gratings to improve pump-light coupling into laser slabs, we calculated the fraction of incident 532-nm pump-light power absorbed by a titanium-doped laser slab. Calculations were performed for the case in which the pumped face has an AR coating that transmits $100 \%$ of the incident pump light, and the case in which the pumped face has a grating of the design described above. For both cases, the back surface was uncoated, pump light was s polarized and the incident angle was $18^{\circ}$. At this angle, we found that the grating splits the incident pump light into $-1,0$ and +1 transmitted orders with intensities relative to the incident intensity of Pump light not absorbed on the first pass through the slab underwent a Fresnel reflection at the back surface and made a second pass. Subsequent reflections of the pump light through the slab were neglected.

Figure 12 shows absorbed fraction vs. the product of absorption coefficient multiplied by slab thickness. Also plotted is the ratio of absorbed fraction with gratings to absorbed fraction without gratings. As the absorption coefficient-thickness product increases from 0 to 3 , the absorption fraction increases from 0 to nearly unity, both with and without gratings. However, between 0 and 3 , there is a significant increase in absorbed fraction when gratings are used. For example, at absorption coefficient-thickness products of 0.5 , 1 and 2 , gratings increase the absorption fraction by $60 \%, 32 \%$ and $10 \%$, respectively.

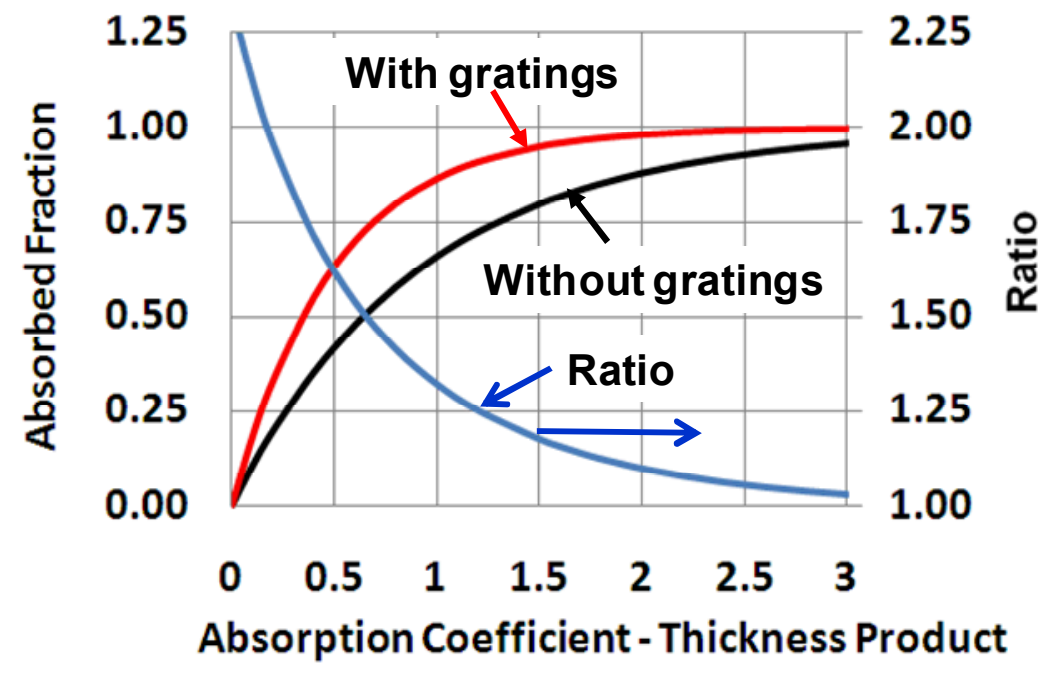

Figure 12. Absorbed fraction of pump light vs. the product of absorption coefficient multiplied by slab thickness, both with gratings and without gratings. Also plotted is the ratio of the absorbed fraction with gratings, to the absorbed fraction without gratings. 


\section{Discussion and Conclusion}

Our calculations show that gratings have the potential for substantially improving the efficiency of titanium-doped sapphire lasers, both by coupling out ASE and by increasing pump-light absorption. Improvements are greatest, however, for designs that are not otherwise optimized for efficiency, either because the pump-pulse duration is long relative to the radiative lifetime so that decay losses are large, or because the dopingthickness product is low so that pump-light is not absorbed efficiently. Since laser diodes remain expensive today, it is often prohibitively expensive to purchase sufficient quantities of diodes to enable solid-state lasers to be pumped on time scales that are short compared with the radiative lifetime. Therefore, diode-pumped solid-state lasers, such as $\mathrm{Nd}$ :glass, Yb:YAG or Yb:S-FAP, are especially strong candidates for the use of gratings to improve efficiency by reducing ASE decay rates. Although the ideal grating groove densities for these materials will be different, we can expect reductions in energy requirements to be similar to values calculated for titanium-doped sapphire, provided similar grating optical properties can be achieved. Yb-doped laser in particular would also benefit from enhanced absorption efficiency offered by gratings, since low iondoping concentrations are desirable for controlling absorption by the thermally-populated lower laser level. Thus, we believe that analysis of grating designs for $\mathrm{Nd}$ :glass, Yb:YAG or $\mathrm{Yb}: \mathrm{S}-\mathrm{FAP}$ is a logical next step in the future development of gratings for laser slabs.

\section{Acknowledgements}

We express our appreciation to Ken Jancaitis at LLNL, who provided us with a copy of his ASE ray-trace code, ASEcalc. 


\section{References}

1) A. C. Erlandson, M. D. Rotter, D. N. Frank and R. W. McCracken, "Design and Performance of the Beamlet Amplifiers," ICF Quarterly Report, Oct-Dec. 1996, UCRL-LR-105821-95-1, Lawrence Livermore National Laboratory, Livermore, California 94551, 1996.

2) A. C. Erlandson, M. Rotter, J. Horvath, D. Frank, R. McCracken, K. Jancaitis, S. Seznec, G. LeTouze, O. Carbourdin, J-C Fornerod, and P. Bosch, "Flashlamppumped Nd:glass amplifiers for the National Ignition Facility," ANS. Fusion Technology 34, 1105-1112, 1998.

3) P. F. Moulton, "Spectroscopic and laser characteristics of Ti:Al2O3", J. Opt. Soc. Am. B 3 (1), 125 (1986) 